\title{
Bacterial Infections and Antibiogram in Device Associated Infections in MICU, ICU of Hospital
}

\author{
Mangala Suresh Harbade, Jyoti Anil Iravane* and Anil A. Gaikwad
}

Department of Microbiology, Government Medical College, Aurangabad, Maharashtra, India

*Corresponding author

\section{Keywords \\ Bacterial infections, Antibiogram, Device associated infections in MICU, ICU}

Article Info

Accepted:

04 February 2019

Available Online:

10 March 2019

\section{A B S T R A C T}

Bacterial infections and antibiogram in device associated infections in MICU, ICU of hospital. The study was carried during the period of Jan 2017 to Dec 2017 in Bacteriology section department of Microbiology in Government Medical College and hospital Aurangabad. 530 samples received from patients of ICU and MICU of hospital for culture and sensitivity. Out of which 330/530 (62\%) samples were from endotracheal secretions suspected of pneumonia and 90/530 (17\%) were Urine samples and 75/530 (14\%) were blood samples. Samples were processed identified by different biochemical reactions. Only device associated hospital acquired infection were studied. Out of 330 samples of ventilated associated pneumonia $199(60 \%)$ showed bacterial growth 131 samples were sterile. In 90 urine samples 34/90 (38\%) samples showed growth and 56 were sterile. In 75 blood samples 22/75 (29\%) samples showed growth and 43 samples were sterile. In this cases pattern of microorganisms were identified with assessment of antibiotic sensitivity profile. The common infection found was ventilated associated pneumonia followed by Urinary tract infection and blood stream infections. Organisms isolated were Klebsiella pneumoniae, Pseudomonas aeruginosa, Acinetobacter baumanii, Escherichia.coli, Enterobacter and Staphylococcus aureus. Gram negative bacteria isolated were in maximum numbers as compared to gram positive cocci. Antibiotic sensitivity pattern of these isolates was done against commonly used antibiotics. Multidrug resistance pattern was noted. Patients admitted in MICU and ICU are more susceptible to Hospital acquired infections. In spite of antibiotic treatment, occurrence of Hospital acquired infections is responsible for increased stay of patients in hospital. Among Hospital acquired infections common Ventilator associated pneumonia, urinary tract infection and blood stream infections. Gram negative bacilli were seen in more numbers than in gram positive cocci. Organisms were showing multidrug resistance pattern. Association of ventilator associated pneumonia were common occurrence followed by Urinary tract infection and blood stream infection. Gram negative bacilli of Enterobactericiae were common isolation. Multidrug resistance to antibiotics was noted. 


\section{Introduction}

Critically ill patients are admitted in Intensive care unit (ICU) and Medical intensive care unit (MICU) of hospital. Bacterial infections are very much common in ICU and MICU of hospital. Patients in ICU have become high risk areas for Hospital acquired infection Sayyeda Gulsitan Siddique et al., (2017). The patients in the ICU and MICU have 5-7 folds higher risk of a Hospital acquired infection than other wards in hospital. Hospital acquired infections are defined as infections acquired in hospital by a patients who was admitted for a reason other than that infection. An infection that are neither present nor incubating when a patients enters hospital Garner et al., (1988).

Hospital acquired infection occurs due to contaminated hospital environment, patients flora, due to severe illness decreased immunity of patients, medical staff and multiple intervention due to invasive device $\mathrm{S}$. Dasgupta et al., (2015).

Patients admitted are on higher antibiotics for longer days in ICU and MICU therefore it is place of multidrug flora. Health Care Worker in ICU is reservoir of common bacterial pathogens which are resistant to antibiotics. Because of this reservoir there is major problem in controlling the infections in ICU. Frequent transfer of patients from wards to ICU and MICU is there in hospital. Concentration of patients who are highly susceptible to infection in one area (intensive care unit) all contribute to development of Hospital acquired infections.

One of the important common Hospital acquired infections is ventilator associated pneumonia (VAP) seen in patients who have been intubated for mechanical ventilation. Since these patients are already critically ill occurrence of VAP is seen in most patients.
Hospital acquired infections can be prevented by taking proper control measures. The study was planned to see the bacterial infections and antibiogram in device associated patients in ICU and MICU. Mainly devise associated infections, ventilator-associated pneumonia (VAP), Catheter associated urinary tract infections, central line blood stream infections were targeted.

\section{Materials and Methods}

The study was carried during the period of Jan 2017 to Dec 2017 in Bacteriology section department of Microbiology in Government Medical College and hospital Aurangabad.

Total numbers of samples received were 530 from patients of ICU and MICU for culture and sensitivity. Out of which 330/530(62\%) samples were from endotracheal secretions suspected of pneumonia and 90/530 (17\%) were suspected of Urine tract infection and 75/530 (14\%) were blood samples.

Patients admitted in hospital for more than 4 days having at least one invasive device (Urinary Catheter, Endotracheal /Tracheostomy tube, Central line) were included.

Patients were having complains fever, increased endotracheal secretion in cases of pneumonia. Most of the patients in Urinary tract infection were catheterized, have fever and increased frequency of urine. Tracheal secretions, urine collected from catheter and blood sample in brain heart infusion broth were received to the Microbiology laboratory.

Grams staining were done. Samples were inoculated on Blood agar and MacConkeys agar. Colonies were read on culture medium after overnight incubations. Organisms were identified from biochemical reactions Collee et al., (1999). 


\section{Results and Discussion}

Total samples 530 were suspected for having Hospital acquired infections. Among the 530 samples the common infection found was ventilator associated pneumonia (330/530$62 \%$ ), followed by urinary tract infections (90/530-17\%) and blood stream infections (75/530-14\%) as shown in Table 1.

Out of 330 samples of ventilated associated pneumonia 199(60\%) showed growth 131 samples were sterile. Klebsiella pneumoniae were isolated in maximum position 90(45\%) followed by Pseudomonas aeruginosa 20(10\%), Acinetobacter baumanii 20 (15\%) and E. coli 20(10\%) and Enterobacter $10(5 \%)$ and Staphylococcus aureus 39(25\%) as shown in Table 2.

In urinary tract infections 90 patients were affected. Out of which 34/90(38\%) samples showed growth and 56 were sterile. Patients showing growth of organism were having catheter. The organisms were Klebsiella pneumoniae (12/90 - 13\%), Pseudomonas aeruginosa (9 /90-10\%), E. coli (9/90-10\%), Staphylococcus aureus (4/90- 4\%). As shown in Table 3. In all above studied infections isolation of gram negative bacteria were in more numbers than gram positive cocci as shown in Table 4.

Blood stream infections were seen in 75patients. Blood cultures of these patients were done. Out of this (22/75-29\%) samples showed growth and 43 samples were sterile. Following organisms were isolated. Klebsiella pneumoniae (8/75-11\%), Pseudomonas aeruginosa $(5 / 75-7 \%)$, E. coli $(2 / 75-3 \%)$ Acinetobacter baumanii (2/75-3\%) and Staphylococcus aureus (5/75-7\%) shown in Table 5. Antibiotic sensitivity testing of all the isolated organisms was done on Muller Hinton agar (MHA) plate by Kirby bauer disc diffusion method using CLSI 2018 guidelines
(5). Readymade antibiotics disc from (Himedia) were used. The following antibiotics used for Gram negative bacteria were Ciprofloxacin (CIP), Ampicillin (AMP), Meropenam (MRP), Amikacin (AMK), piperacillin/tazobactum, Cefotaxime.

Antibiotic disc used for gram positive cocci were Gentamicin(GEN), Erythromycin(ERY), SXT, Linezolid, Tetracycline(TET), Clindamycin, Amoxclav, Cefoxitin.

Resistance to more than one antibiotics were shown by Klebsiella pneumoniae, Pseudomonas aeruginosa and Acinetobacter baumanii in endotracheal aspirate.

In endotracheal aspirate out of 90 (45\%) Klebsiella pneumoniae, 60(67\%) were sensitive to Ampicillin, 40(45\%) were sensitive to CIP, 37(41\%) were sensitive to AMK, 40(45 \%) were sensitive to MRP,60 (67\%) were sensitive to ceftriazone,60 (67\%) were sensitive to CAZ, 90(100\%) were sensitive to colistin. Extended spectrum beta lactase ESBL) producing organisms were 15 in numbers. In ESBLs 10 were males and 5 were females. Out of 20(10\%) Pseudomonas aeruginosa, 18(23\%) were sensitive to pip +taz, 13(14\%) were sensitive to Meropenam and CAZ, 10(11\%) were sensitive to Ciprofloxacin and Gentamycin, 18 (23\%) sensitive to Cetriaxozone.

Out of 39 (25\%) Staphylococcus aureus 13(26\%) MRSA were resistance to Cefoxitin. These were sensitive to Vancomycin and Linezolid. Among the MRSA 9 were males and 4 were females.

\section{Sensitive pattern of antibiotics in Urinary} tract infections is as follows

Out of (12/90-13\%) Klebsiella pneumoniae sensitivity of $10(83 \%)$ were sensitive to Ampicillin, 11(92\%) were sensitive to CIP, 
6(50\%) were sensitive to AMK, 7(58\%) were sensitive to MRP, 11(92\%) were sensitive to Ceftriaxone and CAZ. No ESBL was seen in Urinary tract infections. Out of (9/90-10\%) Pseudomonas aeruginosa, 6(67\%) were sensitive to Pip+Taz, 5(56\%) were sensitive to MRP, 7(78\%) were sensitive to CAZ, 8(90\%) were sensitive to CIP, GEN and Ceftriaxone. Out of E. coli $(9 / 90-10 \%), 8(89 \%)$ were sensitivity to AMP, AMK, GEN, 6(67\%) were sensitive to MRP, 7(78\%) were sensitive to Cefipime, Cefotaxime and Pip+Taz.

Out of Staphylococcus aureus (4 /90- 4\%), $3(75 \%)$ were sensitive to Cefoxitin, TET, GEN, SXT, ERY. 4(100\%) were sensitive to Vancomycin and Linezolid. One was MRSA.

Table.1 Hospital acquired infections in MICU and ICU

\begin{tabular}{|l|l|l|}
\hline Sr. No & HAI in MICU and ICU & Number \& percentage \\
\hline $\mathbf{1}$ & $\begin{array}{l}\text { Ventilator associated } \\
\text { pneumonia }\end{array}$ & $199 / 330=60 \%$ \\
\hline $\mathbf{2}$ & Urinary tract infections & $34 / 90=38 \%$ \\
\hline $\mathbf{3}$ & Blood stream infections & $22 / 75=29 \%$ \\
\hline
\end{tabular}

Table. 2 showing the organisms isolated in Ventilated associated pneumonia

\begin{tabular}{|l|l|ll|}
\hline $\begin{array}{l}\text { Sr.n } \\
\text { o }\end{array}$ & Name of organisms & \multicolumn{2}{|l|}{$\begin{array}{l}\text { Number } \\
\text { percentage }\end{array}$} \\
\hline $\mathbf{1}$ & Klebsiella pneumoniae & 90 & $(45 \%)$ \\
\hline $\mathbf{2}$ & Pseudomonas aeruginosa & 20 & $(10 \%)$ \\
\hline $\mathbf{3}$ & Acinetobacter baumanii & 20 & $(15 \%)$ \\
\hline $\mathbf{4}$ & Enterobacter & 10 & $(5 \%)$ \\
\hline $\mathbf{5}$ & E. coli & 20 & $(10 \%)$ \\
\hline $\mathbf{6}$ & Staphylococcus aureus & 39 & $(25 \%)$ \\
\hline & Total & 199 \\
\hline
\end{tabular}

Table.3 shows organisms isolated in Urinary tract infection

\begin{tabular}{|l|l|ll|}
\hline $\begin{array}{l}\text { Sr.n } \\
\text { o }\end{array}$ & Name of organism & \multicolumn{2}{|l|}{ No. of percentage } \\
\hline $\mathbf{1}$ & Klebsiella pneumonia & $12 / 90$ & $(13 \%)$ \\
\hline $\mathbf{2}$ & Pseudomonas aeruginosa & $9 / 90$ & $(10 \%)$ \\
\hline $\mathbf{3}$ & Escherichia coli & $9 / 90$ & $(10 \%)$ \\
\hline $\mathbf{4}$ & Staphylococcus aureus & $4 / 90$ & $(4 \%)$ \\
\hline & Total & 34 & \\
\hline
\end{tabular}

Table.4 showing percentage of Gram negative bacilli (GNB) and Gram positive cocci (GPC)

\begin{tabular}{|l|l|l|l|}
\hline Sr.no & Name & $\begin{array}{l}\text { Number } \\
(\mathbf{n - 1 9 9 )}\end{array}$ & Percentage \\
\hline $\mathbf{1}$ & Gram negative bacilli & 160 & $80 \%$ \\
\hline $\mathbf{2}$ & Gram positive cocci & 39 & $20 \%$ \\
\hline
\end{tabular}


Table.5 Showing organisms in blood stream infections

\begin{tabular}{|l|l|lc|}
\hline Sr.no & Name of organism & \multicolumn{2}{|c|}{ No. of percentage } \\
\hline $\mathbf{1}$ & Klebsiella pneumonia & $8 / 75$ & $(11 \%)$ \\
\hline $\mathbf{2}$ & Pseudomonas aeruginosa & $5 / 75$ & $(7 \%)$ \\
\hline $\mathbf{3}$ & E. coli & $2 / / 75$ & $(3 \%)$ \\
\hline $\mathbf{4}$ & Acinetobacter baumanii & $2 / 75$ & $(3 \%)$ \\
\hline $\mathbf{5}$ & Staphylococcus aureus & $5 / 75$ & $(7 \%)$ \\
\hline \multicolumn{2}{|c|}{ Total } & 22 & \\
\hline
\end{tabular}

Sensitive pattern of antibiotic in Blood stream infections as follows

Out of (8/75-11\%) Klebsiella pneumoniae, sensitivity pattern of Klebsiella pneumoniae is 4(50\%) were sensitive to Ampicillin, 6(75\%) were sensitive to CIP, 4(50\%) were sensitive to AMK, 3(37\%) were sensitive to MRP, 6(75\%) were sensitive to Ceftriazone, 6(75\%) were sensitive to CAZ.Out of (5/75-7\%) Pseudomonas aeruginosa, sensitivity pattern $4(80 \%)$ were sensitive to pip +taz, 3(60\%) were sensitive to MRP, 4(80\%) were sensitive to CAZ, 5(100\%) were sensitive to CIP, GEN and Ceftriaxone.

Out of (5/75-7\%) samples Sensitivity pattern of Staphylococcus aureus 4(80\%) were sensitive to Cefoxitin, TET, GEN, SXT, ERY. $5(100 \%)$ were sensitive to Vancomycin and Linezolid. In E. coli and Acinetobacter no antibiotic resistance pattern was noted.

Follow up of patients was done by visiting the MICU and ICU wards in Hospital. Patients not responding to treatment were asking to send repeat culture and antibiotics sensitivity was done. Maximum patients were improved.

As the hospital is tertiary care hospital patients are referred from nearby areas, these patients are already given higher antibiotics in private hospitals. The study shows significantly high numbers of infection in ICU and MICU. Risk factors for the development of infections in patients of our study were prior antibiotic administrations. Antibiotic sensitivity testing pattern of the isolated revealed majority are resistant to commonly used antibiotics. The prevention of MICU and ICU acquired infection is must as the pathogens are responsible for severity of infection and increase mortality. In India studies reported occurrence of nosocomial infection in ICU from 11 to $60 \%$ Dasgupta $e t$ al., (2015). The most common hospital acquired infections in our study were Ventilated associated pneumonia followed urinary tract infection and Blood stream infection such similar infection were also noted in Dasgupta et al., (2015) and Vincent et al., (2009).

Most of the ICU acquired infections are caused by gram negative bacilli as seen in Richards et al., (2000).

The most common organism isolated was Klebsiella pneumoniae in three device acquired infection pneumonia, urinary tract infection and blood stream infection which was also studied by Anirban Hom Choudhuri et al., (2017). Involvement of respiratory tract infection and isolation of most common Klebsiella pneumoniae as a pathogen was also noted by previous study done in our same institute Jirwankar PS et al., (2016). Presence of Klebsiella pneumoniae increases the potential risk factors for colonization and infection. Gram negative bacteria were isolated in more numbers than gram positive cocci. 
In late onset VAP gram negative bacteria, MRSA are most common etiological agent. These pneumonia develop after 5 days or more days after initiation of mechanical ventilator in patients. An isolated organism shows multidrug antibiotic resistance which shows increased mortality and morbidity (Guidelines for the management of adult with hospital acquired infections, ventilator associated and health care associated pneumonia (2005).

In conclusion, association of ventilator associated pneumonia were common occurrence followed by Urinary tract infection and blood stream infection. Gram negative bacilli of Enterobactericiae were common isolation. Multidrug resistance to antibiotics was noted. Continuous surveillance and antibiotic stewardship program of hospital is needed.

\section{References}

Anirban Hom Choudhuri,Mitali Chakravarty, and Rajeev Uppal. Epidemiology and characteristics of nosocomial infections in critically ill patients in a tertiary care Intensive Care Unit of Northern India. Saudi J Anaesth. 2017 OctDec; 11(4): 402-407.

American thoracic society, Infectious diseases society of America. Guidelines for the management of adult with hospital acquired infections, ventilator associated and health care associated pneumonia. Am. J Respir Crit Care Med 2005; 23:27-33.

Clinical and laboratory Standards Institute 2016, Performance Standards for Antimicrobial susceptibility testing. Twenty-second information supplement. Wayne PA, USA:
Clinical and laboratory standard institute; 2018.

Collee JG, Miles RS, Watt B. (1999) Tests for the identification of bacteria. In: Collee JG, Fraser AG, Marmion BP, Simmons A, editors. Mackie and McCartney Practical Medical Microbiology. 14th ed. Philadelphia: Churchill Livingstone; pp. 131-49.

Dasgupta, S., Das, S., Chawan, N.S., Hazra, A. (2015). Nosocomial infections in the intensive care unit: Incidence, risk factors, outcome and associated pathogens in a public tertiary teaching hospital of Eastern India. Indian Journal of Critical Care Medicine: Peer-reviewed, Official Publication of Indian Society of Critical Care Med., 19(1):14-20.

Garner JS, Jarvis WR, Emori TG, Horan TC, Hughes JM (1988). CDC definitions for nosocomial infections, 1988. Am J Infect Control. 16:128-40.

P.S. Jirwankar, D. Gambhire. The incidence of devise associated infections, Causative microorganisms and Antibiotic sensitivity pattern in ICU.IOSR Journal of dental and Medical sciences.Vol.15.Issue 8.(2016) pp40.

Richards MJ, Edwards JR, Culver DH, Gaynes RP (2000). Nosocomial infections in combined medical-surgical intensive care units in the United States. Infect Control Hosp Epidemiol. 21:510-5.

Sayyeda Gulsitan Siddique, Manjushree. H. Bhalchandra, Anupama S. Wyawahare, Vishvesh. P. Bansal, Jyotsna. K. Mishra and S. D. Naik (2017). Prevalence of MRSA, ESBL and Carbapenemas Producing isolates obtained from Endotracheal and Tracheal tubes Secretions of ICU Patients at Tertiary care.

Vincent JL, Rello J, Marshall J, Siva E, Anzueto A, Martin CD, et al., (2009). The extended prevalence of infection in the ICU study: EPIC II. JAMA.; 302:2323-9.

\section{How to cite this article:}

Mangala Suresh Harbade, Jyoti Anil Iravane and Anil A. Gaikwad. 2019. Bacterial Infections and Antibiogram in Device Associated Infections in MICU, ICU of Hospital. Int.J.Curr.Microbiol.App.Sci. 8(03): 182-187. doi: https://doi.org/10.20546/ijcmas.2019.803.025 